\title{
Microphone Array Post-Filtering Using Supervised Machine Learning for Speech Enhancement
}

\author{
Pasi Pertilä, Joonas Nikunen \\ Department of Signal Processing, Tampere University of Technology, Finland \\ pasi.pertila@tut.fi, joonas.nikunen@tut.fi
}

\begin{abstract}
High level of noise reduces the perceptual quality and intelligibility of speech. Therefore, enhancing the captured speech signal is important in everyday applications such as telephony and teleconferencing. Microphone arrays are typically placed at a distance from a speaker and require processing to enhance the captured signal. Beamforming provides directional gain towards the source of interest and attenuation of interference. It is often followed by a single channel post-filter to further enhance the signal. Non-linear spatial post-filters are capable of providing high noise suppression but can produce unwanted musical noise that lowers the perceptual quality of the output. This work proposes an artificial neural network (ANN) to learn the structure of naturally occurring post-filters to enhance speech from interfering noise. The ANN uses phase-based features obtained from a multichannel array as an input. Simulations are used to train the ANN in a supervised manner. The performance is measured with objective scores from speech recorded in an office environment. The post-filters predicted by the ANN are found to improve the perceptual quality over delay-and-sum beamforming while maintaining high suppression of noise characteristic to spatial post-filters.

Index Terms:Speech enhancement, Microphone arrays, Array signal processing, Artificial neural networks, Psychoacoustics.
\end{abstract}

\section{Introduction}

Speech enhancement is used to improve the observed quality and it is important in many everyday applications such as telephony and distant talking interfaces. When the talker is distant from the capturing microphone, reverberation and background noise often reduce the captured quality significantly. Speech enhancement can remove noise (denoising), reverberation (dereverberation), or both. When multiple speakers are talking concurrently the problem of removing the interfering speakers is called speech separation.

Time-frequency (T-F) masking is based on the windowingdisjoint orthogonality assumption of signals, i.e. speech energy is concentrated only to few time-frequency points, which do not overlap between speakers [1]. A T-F mask typically approximates the ideal binary mask (IBM) and is applied by multiplying the observed mixture, thus passing only the desired components. However, musical noise artifacts can arise due to errors in mask estimation. Recently, the real-valued ideal Wiener filter (IWF) has been shown to improve speech intelligibility in noisy conditions over IBM [2].

Machine learning techniques are popular in speech enhancement. In [3] a non-negative matrix factorization (NMF) technique is used to learn spectral basis of speech and different

\footnotetext{
${ }^{*}$ Research work funded by Finnish Academy project no. 138803.
}

noise types. The NMF reconstruction is then used to denoise the observation. The authors of [4] train a long short-term memory (LSTM) recurrent neural network (RNN) to predict a T-F mask for speech enhancement. In [5] spectral features (such as Melfrequency cepstral coefficients) and their delta components are used to train a deep neural network (DNN) to predict the instantaneous SNR for each frequency band, which is used to estimate the ideal ratio mask IRM. The authors of [6] use a combination of DNNs and support vector machines (SVMs) for speech enhancement by binary classification of T-F bands. In [7], deep recurrent autoencoder neural network is trained to denoise input features for noise robust automatic speech recognition (ASR).

While the above methods primarily utilize a monophonic signal, binaural signals enable the use of spatial cues, i.e., interaural time delay (ITD) and interaural level difference (ILD). The degenerate unmixing estimation technique (DUET) clusters each TF point based on its cue values [8]. In [9] this is done by supervised learning via kernel-density estimation for a binary T-F mask value. In [10] the spatial cues (along with pitch features for voiced frames) are used to train two sets of multilayer perceptrons (MLPs) for each combination of azimuth angle and frequency band. This approach requires a lot of training data and computations.

Beamforming is linear filtering applied to microphone array signals in order to amplify the desired direction(s) and/or attenuate unwanted one(s). The most simple fixed weight beamformer is the delay-and-sum beamformer (DSB) that sums the temporally aligned input signals from the desired direction of arrival (DOA). In contrast, adaptive methods update the filter coefficients based on estimates of the noise and signal statistics. The beamforming output can be further enhanced by multiplying with a post-filter, i.e. a type of T-F mask. An adaptive beamformer known as minimum variance distortionless response (MVDR) combined with the single channel Wiener filter has been shown to be an optimal approach in the minimum mean square error (MMSE) sense [11, Ch.3]. The ability to increase the SNR of the beamformer output has been successfully shown with different post-filters [12, 11, 13, 14], which differ in the assumptions made of the signal and noise.

A spatial post-filter can suppress also point-wise noise sources. Tashev et al. derived the instantaneous DOA (IDOA) filter in [15], in which phase-difference measurements form a likelihood function for post-filter estimation. Selzer et al. [16] proposed a statistical generative model to estimate speech and noise parameters as Gaussian random variables with application to post-filtering using phase-difference and spectral observations for a four microphone linear array. As in [10] the phase based features are dependent on the angle of the source. While spatial filtering has impressive suppression of noise as evident in [15] it can also produce unwanted artifacts that lead to lower 
perceptual quality than that of the simple DSB. Therefore, it is important to investigate noise suppression capability of spatial filtering in conjunction with perceptual quality. Selzer et al. [17] proposed a log-MMSE adaptive beamformer that uses the spatially post-filtered signal as the desired signal to produce higher perceptual quality over DSB.

This work proposes the use of a multilayer perceptron (MLP), a type of artificial neural network, to learn the mapping from phase-based features directly into post-filter values using a circular microphone array. In contrast to angle dependent models $[16,10]$ the input feature is angle independent and a single MLP can be used to predict the post-filter. This reduces the model complexity over previous methods. In contrast to previous binaural approaches that utilize the IBM as the target, the MLP here predicts the IWF, i.e., a real-valued postfilter. Finally, in contrast to traditional post-filters, the MLP does not require explicit assumptions or estimates of the signal and noise statistics. Instead, data generated by simulations is used to train the MLP, while the performance is evaluated with recorded speech. The proposed MLP based post-filter operates in the MEL-frequency domain.

This paper is organized as follows. Section 2 reviews beamforming and DOA estimation. The conventional spatial postfilter is reviewed in Section 3. The proposed MLP based spatial post-filter is presented in Section 4. Section 5 describes the array speech recordings. Section 6 reports and discusses the results and is followed by the conclusions Section 7 .

\section{Beamforming and DOA Estimation}

The $i$ th microphone signal $x_{i}(t), i=1, \ldots, M$ is modeled as delayed and attenuated version of the source signal $s(t)$ embedded in additive noise $v_{i}(t)$. Using discrete time short-time Fourier transform the $i$ th input signal is

$$
X_{i}(t, \omega)=H_{i}(t, \omega) S(t, \omega)+V_{i}(t, \omega),
$$

where $H_{i}(t, \omega)$ is the transfer function between the $i$ th microphone and source, $\omega$ is angular frequency, and $t$ is time frame index. The delay-and-sum beamformer (DSB) with post-filtering aligns the microphone signals before summation

$$
Y(t, \omega)=\underbrace{\frac{1}{M} \sum_{i=1}^{M} X_{i}(t, \omega) e^{j \omega \tau_{i, 1}(\mathbf{k})}}_{Y_{\mathrm{DSB}}(t, \omega)} \cdot H_{\text {post }}(t, \omega)
$$

where $H_{\text {post }}(t, \omega)$ is the real-valued post-filter gain. The steering delays are time difference of arrival (TDOA) values defined as

$$
\tau_{i, j}(\mathbf{k})=\left(\mathbf{m}_{i}-\mathbf{m}_{j}\right)^{\mathrm{T}} \mathbf{k},
$$

where $\mathbf{m}_{i} \in \mathbb{R}^{3 \times 1}$ denotes the $i$ th microphone position, and $\mathbf{k} \in \mathbb{R}^{3 \times 1}$ denotes the source emitted wavefront's direction of arrival (DOA) with respect to array origin. Both vectors are represented in a Cartesian coordinate system. The relation to spherical coordinates (azimuth $\theta$ and elevation $\phi$ ) is

$$
\mathbf{k}=c^{-1}[\sin (\theta) \cos (\phi), \cos (\theta) \cos (\phi), \sin (\phi)]^{\mathrm{T}},
$$

where $c$ is the speed of sound, and consequently $\|\mathbf{k}\|=c^{-1}$, where $\|\cdot\|$ denotes Euclidean norm.

\subsection{DOA estimation}

The generalized cross-correlation (GCC) is applied to estimate the source DOA $\mathbf{k}$ in frame $t$ with the steered response power (SRP) method [18]
$R_{\mathrm{gcc}}(t, \mathbf{k})=\sum_{\forall\{i, j\}} \int_{-\pi}^{\pi} \psi_{i j}(t, \omega) X_{i}(t, \omega) X_{j}^{*}(t, \omega) e^{j \omega \tau_{i, j}(\mathbf{k})} d \omega$,

where the summation is over all unique pairs $\{i, j\}$, $\begin{gathered}(5) \\ \text { and }\end{gathered}$ $\psi_{i j}(t, \omega)=\left|X_{i}(t, \omega) X_{j}^{*}(t, \omega)\right|^{-1}$ is PHAT weighting that removes the amplitude information. For simplicity, a static source is assumed, and a finite grid of fixed DOA vectors $\mathbf{K}=\left[\mathbf{k}_{1}, \ldots, \mathbf{k}_{N}\right]^{\mathrm{T}}$ is used to evaluate a log-energy weighted response over time. The maximum weight DOA vector is extracted as the DOA estimate

$$
\hat{\mathbf{k}}=\underset{\mathbf{k} \in \mathbf{K}}{\operatorname{argmax}}\left\{\sum_{t=0}^{T} E(t) \cdot R_{\mathrm{gcc}}(t, \mathbf{k})\right\},
$$

where $E(t)=10 \log _{10}\left(\frac{1}{M} \sum_{i=1}^{M} \int_{-\pi}^{\pi}\left|X_{i}(t, \omega)\right|^{2} d \omega\right)$ is the log-energy of frame $t$, and $T$ is the number of frames.

\section{Conventional Spatial Post-Filter}

Following the azimuth angle IDOA filter definition of [15] and omitting the time index for brevity the expression of IDOA for a DOA vector $\mathbf{k}$ is

$$
\Upsilon_{\omega}(\mathbf{k})=\frac{\left\|\boldsymbol{\Delta}(\omega)-\Psi_{\omega}(\mathbf{k})\right\|}{\left\|\frac{\partial \Psi_{\omega}(\mathbf{k})}{\partial \mathbf{k}}\right\|},
$$

where $\boldsymbol{\Delta}(\omega) \in \mathbb{R}^{P \times 1}$ is a vector of measured pairwise phase differences between all $P=M(M-1) / 2$ unique microphone pairs $\{i, j\}$, with elements

$$
\boldsymbol{\Delta}(\omega)=\left[\angle X_{1}(\omega)-\angle X_{2}(\omega), \ldots, \angle X_{M-1}(\omega)-\angle X_{M}(\omega)\right]^{\mathrm{T}},
$$

where $\angle(\cdot)$ is the phase angle of a complex number. The theoretical phase difference vector is defined as

$$
\Psi_{\omega}(\mathbf{k})=\omega \cdot\left[\tau_{1,2}(\mathbf{k}), \tau_{1,3}(\mathbf{k}), \ldots, \tau_{M-1, M}(\mathbf{k})\right]^{\mathrm{T}},
$$

where $\Psi_{\omega}(\mathbf{k}) \in \mathbb{R}^{P \times 1}$. Finally, $\partial \Psi_{\omega}(\mathbf{k}) / \partial \mathbf{k}$ is the partial derivative of $\Psi_{\omega}(\mathbf{k})$ with respect to $\mathbf{k}$.

The probability density for frequency $\omega$ to come from desired direction $\mathbf{k}$ is [15]

$$
p(\omega, \mathbf{k})=\frac{1}{\sqrt{2 \pi \lambda_{\omega}(\mathbf{k})}} \exp \left(-\frac{\Upsilon_{\omega}(\mathbf{k})^{2}}{2 \lambda_{\omega}(\mathbf{k})}\right),
$$

where $\lambda_{\omega}(\mathbf{k})$ is spatial variance estimated from data [15]. The direct spatial post-filter (DSPF) is [15]

$$
H_{c}(t, \omega)=\frac{p(\omega, \mathbf{k})}{\sum_{l=1}^{L} p\left(\omega, \mathbf{k}_{l}\right)},
$$

where $\mathbf{k}_{l}$ denotes $l=1, \ldots, L$ different steering directions. DSPF allows steep noise suppression but entails artifacts. Note that [15] proposes the additional use of a HMM framework.

\section{Neural Network Based Post-Filter}

A block diagram of beamforming with spatial post-filtering is presented in Fig. 1. The post-filter values are obtained in the MEL frequency domain. A widely applied conversion from linear frequency $f_{\mathrm{Hz}}$ (in $\mathrm{Hz}$ ) to MEL frequency is

$$
\operatorname{MEL}\left(f_{\mathrm{Hz}}\right)=2595 \log _{10}\left(f_{\mathrm{Hz}} / 700+1\right) .
$$

The use of MEL-frequency scale is motivated by the psychoacoustic properties of the human hearing system i.e. closely spaced frequencies mask each other. Furthermore, the computational complexity of post-filter gain for $B$ frequency bands instead of $N_{\text {DFT }}$ frequency bins can lead to large computational savings, since typically $B \ll N_{\text {DFT }}$. 


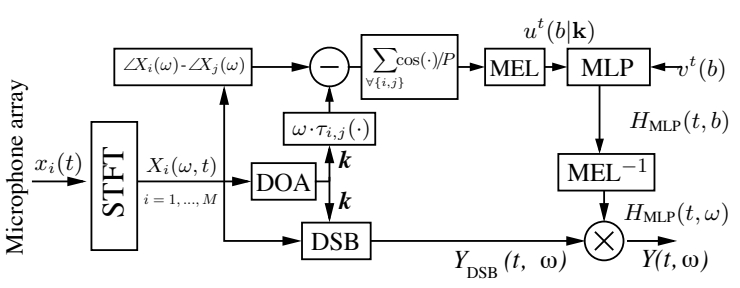

Figure 1: A block diagram of the proposed post-filter approach. The phase-differences are first extracted between microphone pairs, then subtracted from theoretical delays, and converted into input features $u^{t}(b \mid \mathbf{k})$ for frequency bands $b=1, \ldots, B$. Similarly, averaged features over other directions are extracted as $v^{t}(b)$. Using these values, the MLP predicts the post-filter values for each frequency band. The frequency band values are then converted to linear scale $H_{\mathrm{MLP}}(t, \omega)$. Finally, post-filter values are applied to the beamformer output $Y_{\mathrm{DSB}}(t, \omega)$.

\subsection{MLP Structure}

Given an estimated look direction $\mathbf{k}(6)$, a spatial feature $u^{t}(\omega \mid \mathbf{k})$ is calculated by averaging the cosines of differences between the measured and theoretical phase differences over all $P$ microphone pairs $\{i, j\}$

$$
u^{t}(\omega \mid \mathbf{k})=\frac{1}{P} \sum_{\forall\{i, j\}} \cos \left(\angle X_{i}(t, \omega)-\angle X_{j}(t, \omega)-\omega \tau_{i, j}(\mathbf{k})\right),
$$

where $u^{t}(\omega \mid \mathbf{k}) \in[-1,+1]$ is a scalar value at time $t$. Note that the $\cos (\cdot)$ function is used to wrap the phase values into the range $[-1,+1]$, and therefore value $u^{t}(\omega \mid \mathbf{k})=1$ indicates perfect agreement between measured and theoretical phase differences between all microphone pairs. The frequency features are then transferred into $b=1, \ldots, B$ MEL band values $u^{t}(b \mid \mathbf{k})$.

The information about signals arriving from non-look directions is taken from the average value over non-look direction feature values

$$
v^{t}(b)=\frac{1}{L} \sum_{l=1}^{L} u^{t}\left(b \mid \mathbf{k}_{l}\right),
$$

where direction vectors $\mathbf{k}_{1}, \ldots, \mathbf{k}_{L}$ are spread around the space, and do not include the desired direction. The vector

$$
\mathbf{f}^{t}=\left[u^{t}(1 \mid \mathbf{k}), \ldots, u^{t}(B \mid \mathbf{k}), v^{t}(1), \ldots, v^{t}(B)\right]^{\mathrm{T}}
$$

is the input vector of the MLP. The size of the input layer is therefore $2 B$ (excluding the bias).

One hidden layer twice the size of the input layer was selected empirically. The $B$ output layer values are assigned as the post-filter values in the MEL frequency domain, i.e. $H_{\mathrm{MLP}}(t, b)=o_{b}^{t}\left(\mathbf{f}^{t}, \mathbf{w}\right)$, where $b=1, \ldots, B$. The value of the $b$ th node of the output layer can be written as [19]

$$
o_{b}^{t}\left(\mathbf{f}^{t}, \mathbf{w}\right)=\sigma\left(\sum_{j=1}^{4 B} w_{b j}^{(2)} \sigma\left(\sum_{i=1}^{2 B} w_{j i}^{(1)} f_{i}^{t}+w_{j 0}^{(1)}\right)+w_{b 0}^{(2)}\right),
$$

where $w_{j i}^{(1)}$ denotes the weight given for the $i$ th input by hidden layer neuron $j, w_{b j}^{(2)}$ is the weight given for the output of the $j$ th hidden layer neuron by the $b$ th node of the output layer, $\sigma(\cdot)$ is the logistic sigmoid function, $w_{j 0}^{(1)}$ and $w_{b 0}^{(2)}$ are bias weights, and $\mathbf{w}$ simply denotes the set of all weights. Note that the logistic function is also applied at the output nodes, which bounds the post-filter value between the range $[0,1]$.

The linear domain post-filter values are obtained by inverse mapping denoted as $\mathrm{MEL}^{-1}$. Figure 1 represents a block diagram of the process.
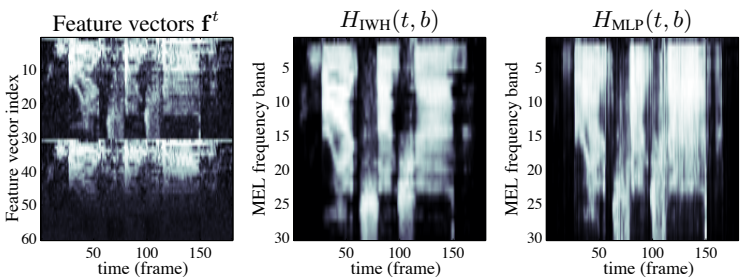

Figure 2: Left panel displays input feature vectors $\mathbf{f}^{t}$ at each time frame $t$. Middle panel is the target value (ideal Wiener filter) in MEL frequency domain. Right panel displays the predicted values in MEL frequency domain.

\subsection{Training Data}

An eight microphone circular array with $10 \mathrm{~cm}$ radius was used to simulate audio with $16 \mathrm{kHz}$ sampling rate with added noise and reverberation. Two different sized rooms with reverberation times $\left(\mathrm{T}_{60}\right) 0.4 \mathrm{~s}$ and $0.9 \mathrm{~s}$ with two source distances of $1.2 \mathrm{~m}$ and $2.4 \mathrm{~m}$ were used to generate room impulse responses (RIR) for each microphone using the image method [20]. For each room and distance combination 100 randomly selected TIMIT database speech sentences were convolved with the RIRs to simulate the reverberant array signals. In each repetition, the array was placed in the center of the room, and the source angle was drawn randomly between surrounding azimuth angles $\left[0^{\circ}, 360^{\circ}\right]$. Independent and identically distributed white Gaussian noise was added to the microphone signals, and the resulting SNR was drawn from a uniform distribution between $[+12,+40] \mathrm{dB}$. The purpose of adding noise is to provide diverse training samples for the neural network in order to be generic enough to be applied in different conditions.

A $32 \mathrm{~ms}$ window with $75 \%$ overlap was used to extract the features (15) from all 400 simulated recordings. The target values were obtained from the ideal Wiener filter (IWF) [21]

$$
H_{\mathrm{IWF}}(t, \omega)=\frac{P_{s s}(t, \omega)}{P_{s s}(t, \omega)+P_{n n}(t, \omega)},
$$

where $P_{s s}(t, \omega)$ and $P_{n n}(t, \omega)$ are the signal and noise power spectra. In the IWF evaluation $P_{s s}(t, \omega)$ contains only the direct path component of the impulse response convolved with the talker's signal. Early reflections and late reverberation components arrive from different directions than the source and are omitted, since the combined phase difference measurement (13) can only be related to one direction. $P_{n n}(t, \omega)$ contains the added noise. The data was divided into $80 \%$ training, $10 \%$ testing, and $10 \%$ evaluation sets. The error back-propagation algorithm was used to train ${ }^{1}$ MLP weights $\mathbf{w}$ (16) using stochastic gradient descent using mini-batches of 100 samples. The training was stopped when the performance on the test set did not improve in 15 epoch or epochs exceeded 500. After 500 epochs, the training was stopped and the MSE on evaluation data was 0.0157 . Figure 2 displays an example of the input vectors (left panel), the desired IWF values (middle panel), and the predicted values (right panel). It is noted that the upper part of the feature vector, i.e. values $f_{1}, \ldots, f_{B}$, could be used to predict the T-F mask as such. However, including the latter part of the feature vector $f_{B+1}, \ldots, f_{2 B}$, was found to discriminate against spatial noise sources.

${ }^{1}$ The Deep Learn Toolbox implementation of MLP
was used, http://github.com/rasmusbergpalm/
DeepLearnToolbox.




\section{Description of Recordings}

An small office was used to capture speech recordings with an 8 channel microphone array with $10 \mathrm{~cm}$ radius and a reference microphone mounted on a stand at $1.5 \mathrm{~m}$ height. The array was elevated on a stand at $1.0 \mathrm{~m}$ height, and consisted of omnidirectional electret condenser microphones (Sennheiser MKE 2). The reference microphone was a cardioid pattern Røde NT 55 condenser microphone. The recordings consist of phonetically balanced sentences [22] captured at $1.3 \mathrm{~m}$ (near) and $2.0 \mathrm{~m}$ (far) distance from the array center with $48 \mathrm{kHz}$ sampling rate. Two PCs were emitting fan noise at approximately $1 \mathrm{~m}$ and $1.5 \mathrm{~m}$ distances from the array, in different angles than the speaker. A total of 77 recordings were captured from four different male speakers (38 far, 39 near), with an average sentence length of $3.8 \mathrm{~s}$.

\section{Results and Discussion}

The number of MEL-frequency bands was empirically set to $B=30$. The data was downsampled to $16 \mathrm{kHz}$, and processed in $32 \mathrm{~ms}$ Hann weighted windows with $75 \%$ overlap between sequential frames. The predefined look directions for DOA estimation $\mathbf{K}$ were distributed to the upper hemisphere of the array, refer to Eq. (6). Ten different $(L=10)$ directions were used to evaluate the features from other directions, refer to Eq. (14).

\subsection{Objective Metrics}

The objective performance in terms of segmental SNR was measured using the RMS normalized reference signal $S(t, f)$ and the RMS normalized enhanced signal $Y(t, f)$

$$
\mathrm{SNR}=\frac{10}{T \cdot N_{\mathrm{DFT}}} \sum_{t=1}^{T} \sum_{f=1}^{N_{\mathrm{DFT}}} \log _{10} \frac{|S(t, f)|^{2}}{(|S(t, f)|-|Y(t, f)|)^{2}}
$$

where $f$ is discrete frequency index, and $N_{D F T}=512$. The frequency-weighted segmental SNR (fwSNRseg) was also evaluated since it has been reported to have a high correlation with perceptual quality $[23]^{2}$.

A short-time objective intelligibility measure (STOI) [24] is used to evaluate the perceptual quality of speech. It is designed for predicting the intelligibility of TF-weighted noisy speech.

\subsection{Results}

The results with the discussed metrics over the recordings are presented in Table 1. Both distances are reported separately. Scores for four methods are reported: a single microphone signal of the array, DSB, DSB with DSPF (11), and DSB with the the proposed MLP based post-filter (described in Sec. 4).

When the reference microphone is in the near location (1.3 $\mathrm{m}$ from array center) the STOI score for the single microphone is 0.72 and is increased to 0.79 by using DSB. The utilization of DSB also adds $3 \mathrm{~dB}$ to the fwSNRseg, and $4 \mathrm{~dB}$ to the SNR in contrast to using a single microphone. By using the proposed MLP post-filter on the DSB output, the STOI score is further increased to 0.84 . Furthermore, the SNR and the fwSNRseg are both increased by approximately $5 \mathrm{~dB}$ over DSB. This shows a clear improvement for the proposed postfilter over DSB in both the capability to suppress noise. The DSB+DSPF has lower STOI score than DSB, but has noise suppression over DSB. This is in line with previous research [25] that reported PESQ [26] values for the speech quality.

\footnotetext{
${ }^{2}$ Implemented with 23 MEL-frequency bands, and $\gamma=0.2$
}

Table 1: Average scores of each method under test, i.e., a single array microphone (Mic.), delay-and-sum beamforming (DSB), DSB with a direct spatial post-filter (DSB+DSPF), and DSB with the proposed MLP based post-filter (DSB+MLP). Mean scores of two talker distances are given.

\begin{tabular}{|l|cccc|}
\hline Near $(1.3 \mathrm{~m})$ & Mic. & DSB & DSB+DSPF & DSB+MLP \\
\hline STOI & 0.72 & 0.79 & 0.76 & $\mathbf{0 . 8 4}$ \\
fwSNRseg $(\mathrm{dB})$ & -0.6 & 2.3 & 5.5 & $\mathbf{6 . 8}$ \\
SNR $(\mathrm{dB})$ & -4.0 & -0.1 & 4.3 & $\mathbf{4 . 9}$ \\
\hline \hline Far $(2.0 \mathrm{~m})$ & Mic. & DSB & DSB+DSPF & DSB+MLP \\
\hline STOI & 0.65 & 0.71 & 0.72 & $\mathbf{0 . 7 8}$ \\
fwSNRseg $(\mathrm{dB})$ & -0.4 & 1.4 & 4.2 & $\mathbf{5 . 1}$ \\
SNR $(\mathrm{dB})$ & -3.9 & -0.9 & 3.2 & $\mathbf{3 . 9}$ \\
\hline
\end{tabular}

In the more difficult scenario the reference microphone is at $2.0 \mathrm{~m}$ distance from the array. The overall scores for the single microphone signal do not decrease much despite the increase in talker distance. However, all DSB metrics are lower than in the near position. Once again, the use of MLP post-filter on the DSB increases the STOI by 0.07 to 0.78 , fwSNRseg and SNR are increased approximately by $4 \mathrm{~dB}$ and $5 \mathrm{~dB}$. The increase in scores when using the proposed MLP post-filter is almost the same as in the near condition. Similarly the to near condition, in the far condition the DSB+DSPF has STOI score near DSB, but shows large noise suppression over DSB

The results with the gathered speech data show that the proposed method increases both the perceptual quality (in terms of intelligibility) and suppression of noise over DSB. The MLP training did not include any samples from the recording environment, and utilized only simulations with the TIMIT database speech samples. This means that the MLP was successful in generalizing the prediction of the post-filter in an unseen environment, which increases the method's practical value. The DOA estimation was kept as simple as possible, and did not consider moving sources. We note that the MLP prediction utilizes only the most recent input feature vector. Therefore, the approach is applicable with any source direction tracking method. In the future, we would like to extend the method towards separating multiple moving talkers, and evaluate the effect of using more advanced neural network structures.

\section{Conclusions}

This paper proposes using an artificial neural network (ANN) in the design of spatial post-filtering for beamforming. More specifically, the multilayer perceptron (MLP) is applied. Spatial cues from noisy and reverberant speech are used to train a MLP to predict post-filter values corresponding to the ideal Wiener Filter (IWF). The post-filter is obtained in the MEL-frequency scale and is converted to linear frequency scale before being applied to delay-and-sum beamforming (DSB). The method was evaluated with microphone array recordings of speech sentences in an office at two different distances. Objective measurements of intelligibility (STOI) show that the MLP based post-filter provides increase in perceptual quality over DSB, while the segmental SNR and frequency-weighted segmental SNR indicate significant noise suppression over DSB.

\section{References}

[1] DeLiang Wang, "Time-Frequency Masking for Speech Separation and its Potential for Hearing Aid Design," Trends in Amplification, vol. 12, pp. 332-353, 2008.

[2] N. Madhu, A. Spriet, S. Jansen, R. Koning, and 
J. Wouters, "The Potential for Speech Intelligibility Improvement Using the Ideal Binary Mask and the Ideal Wiener Filter in Single Channel Noise Reduction Systems: Application to Auditory Prostheses," IEEE Transactions on Audio, Speech, and Language Processing, vol. 21, no. 1, pp. 63-72, Jan 2013.

[3] K. Wilson, B. Raj, P. Smaragdis, and A. Divakaran, "Speech Denoising Using Nonnegative Matrix Factorization with Priors," in Proc. IEEE International Conference on Acoustics, Speech, and Signal Processing (ICASSP), 2008, pp. 4029-4032.

[4] F. Weninger, F. Eyben, and B. Schuller, "Single-Channel Speech Separation With Memory-Enhanced Recurrent Neural Networks," in Proc. IEEE International Conference on Acoustics, Speech, and Signal Processing (ICASSP), 2014.

[5] A. Narayanan and DeLiang Wang, "Ideal Ratio Mask Estimation Using Deep Neural Networks for Robust Speech Recognition," in Proc. IEEE International Conference on Acoustics, Speech and Signal Processing (ICASSP), 2013.

[6] Yuxuan Wang and DeLiang Wang, "Towards Scaling Up Classification-Based Speech Separation," IEEE Transactions on Audio, Speech, and Language Processing, vol. 21, no. 7, pp. 1381-1390, July 2013.

[7] A. L. Maas, Q. V. Le, T. M. O’Neil, O. Vinyals, P. Nguyen, and A. Y. Ng, "Recurrent Neural Networks for Noise Reduction in Robust ASR," in Proc. 13th Annual Conference of the International Speech Communication Association (Interspeech), 2012.

[8] Özgür Ylmaz and Scott Rickard, "Blind Separation of Speech Mixtures via Time-Frequency Masking," IEEE Transactions on Signal Processing, vol. 7, no. 52, pp. 1830-1847, July 2004.

[9] N. Roman, D. Wang, and G. J. Brown, "Speech Segregation based on Sound Localization," J. Acoust. Soc. Am., vol. 114, no. 4, pp. 2236-2252, 2003.

[10] J. Woodruff and DeLiang Wang, "Binaural Detection, Localization, and Segregation in Reverberant Environments based on Joint Pitch and Azimuth Cues," IEEE Transactions on Audio, Speech, and Language Processing, vol. 21, no. 4, pp. 806-815, 2013.

[11] M. Brandstein and D. Ward, Eds., Microphone Arrays, Springer-Verlag, 2001.

[12] R. Zelinski, "A Microphone Array with Adaptive PostFiltering for Noise Reduction in Reverberant Rooms," in Proc. IEEE International Conference on Acoustics, Speech, and Signal Processing (ICASSP), 1988.

[13] I.A. McCowan and H. Bourlard, "Microphone Array PostFilter based on Noise Field Coherence," IEEE Transactions on Speech and Audio Processing, vol. 11, no. 6, pp. 709-716, Nov 2003.

[14] S. Lefkimmiatis and P. Maragos, "A Generalized Estimation Approach for Linear and Nonlinear Microphone Array Post-Filters," Speech communication, vol. 49, no. 7, pp. 657-666, 2007.

[15] I. Tashev and A. Acero, "Microphone Array PostProcessor Using Instantaneous Direction of Arrival," Proc. Int. Workshop on Acoustic Echo and Noise Control (IWAENC), 2006
[16] M.L. Seltzer, I. Tashev, and A. Acero, "Microphone Array Post-Filter using Incremental Bayes Learning to Track the Spatial Distributions of Speech and Noise," in Proc. IEEE International Conference on Acoustics, Speech and Signal Processing (ICASSP), 2007.

[17] Michael L. Seltzer and Ivan Tashev, "A Log-MMSE Adaptive Beamformer Using a Nonlinear Spatial Filter," in Proc. 11th Int. Workshop on Acoustic Echo and Noise Control (IWAENC), 2008.

[18] J. H. DiBiase, H. F. Silverman, and M. S. Brandstein, "Robust Localization in Reverberant Rooms," in Microphone Arrays, M. Brandstein and D. Ward, Eds., chapter 8, pp. 157 - 180. Springer-Verlag, 2001.

[19] C.M. Bishop, Pattern Recognition and Machine Learning, Information Science and Statistics. Springer, 2006.

[20] J. Allen and D. Berkley, "Image Method for Efficiently Simulating Small-Room Acoustics," J. Acoust. Soc. Am. vol. 65, no. 4, pp. 943 - 950, 1979.

[21] E. Diethorn, "Subband Noise Reduction Methods for Speech Enhancement," in Audio Signal Processing for Next-Generation Multimedia Communication Systems, Y.(A.) Huang and J. Benesty, Eds., chapter 4. Kluwer Academic Publishers, 2004.

[22] "IEEE Recommnded Pratice for Speech Quality Measurements," Audio and Electroacoustics, IEEE Transactions on, vol. 17, no. 3, pp. 225-246, Sep 1969.

[23] Yi Hu and P.C. Loizou, "Evaluation of objective quality measures for speech enhancement," IEEE Transactions on Audio, Speech, and Language Processing, vol. 16, no. 1, pp. 229-238, Jan 2008.

[24] C. H. Taal, R. C. Hendriks, R. Heusdens, and J. Jensen, "An Algorithm for Intelligibility Prediction of TimeFrequency Weighted Noisy Speech," IEEE Transactions on Audio, Speech, and Language Processing, vol. 19, no. 7, pp. 2125-2136, 2011.

[25] Ivan J. Tashev, Sound Capture and Processing: Practical Approaches, John Wiley \& Sons Ltd., 2009.

[26] "Perceptual evaluation of speech quality (PESQ): An objective method for end-to-end speech quality assessment of narrow-band telephone networks and speech codecs," in ITU-T, P.862, SERIES P: TELEPHONE TRANSMISSION QUALITY, TELEPHONE INSTALLATIONS, LOCAL LINE NETWORKS. Methods for objective and subjective assessment of quality. 02/2001. 